\title{
Cognitive Remediation Program for Family Caregivers in Schizophrenia (CIRCUS): Study of the Effectiveness of a Guided AND WEB-BASED Compensatory Cognitive Remediation: A Randomized Pilot Study
}

Amandine Garbisson ( $\nabla$ a-garbisson@chu-montpellier.fr )

Hospital La Colombiere: Hopital La Colombiere https://orcid.org/0000-0002-2830-8272

Yasmine Laraki

Hôpital La Colombière: Hopital La Colombiere

Tifany Dubois

Hôpital La Colombière: Hopital La Colombiere

Myrtille André

Hôpital La Colombière: Hopital La Colombiere

Nicolas Rainteau

Hôpital La Colombière: Hopital La Colombiere

Delphine Capdevielle

Hôpital La Colombière: Hopital La Colombiere

Stéphane Raffard

Universite Paul-Valery Montpellier 3

\section{Research Article}

Keywords: Schizophrenia, cognitive remediation, family, web-based therapy

Posted Date: July 12th, 2021

DOl: https://doi.org/10.21203/rs.3.rs-561341/v1

License: (c) (i) This work is licensed under a Creative Commons Attribution 4.0 International License.

Read Full License 


\section{Abstract}

\section{Background}

Cognitive impairments are extremely common in schizophrenia and strongly predict deficits in daily functioning, poor management of medication, and multiple hospitalizations. Cognitive remediation is recognized to have a positive impact on cognitive impairments by engaging preserved cognitive functions or by implementing environmental supports that sustain independent living.

Velligan et al. [1] developed and tested a manualized intervention, called Cognitive Adaptation Training (CAT). In this program, trained mental health specialists implemented compensatory techniques such as environmental supports in the individual's living environment in order to live more independently and achieve greater self-sufficiency.

However, implementing this program requires a lot of professionals and time to maintain CAT effect. This type of intervention is not widely available in community care which may explain the large number of patients who are dependent on family members for daily living activities. Training family members in this form of intervention would be an appropriate way to resolve these issues. Family expressed a real interest in these types of home-support strategies that CAT offers. Recently, Kidd et al. [2] developed a CAT version for Families and created a manual accessible to people without any knowledge of cognitive deficits. This manual helps families to select specific cognitive-adaptative strategies with their relative to achieve targeted goals. This method has been translated in French.

The aim of this study is to examine whether Web-based Family Cognitive Adaptation Training can improve functioning, medication adherence and negative symptoms for individuals with schizophrenia as well as reduce burden for the family members.

\section{Methods/Design}

A total of 60 Dyads consisting of one caregiver and one supported individual with schizophrenia will be randomized to either Web-based family cognitive adaptation training or an internet-based control condition (psycho-education). The primary outcome measure will be the total score on the life skills profile. Secondary outcome measures will include the global score of the Zarit burden Interview, the PANSS negative score, the CAINS score, patient medication adherence, and patient and caregiver quality of life.

\section{Discussion}

We hope that this type of intervention could be developed in territorial areas where professionals are not trained to cognitive remediation and therefore substantially lower the barrier to the deployment of cognitive interventions with other psychosocial interventions for individuals with schizophrenia and their caregivers. 
Trial registration: ClinicalTrials.gov Identifier: NCT04173598. Registered on November 22th 2019

\section{Introduction}

\section{Background}

Cognitive impairments are extremely common in schizophrenia [3,4], affecting 70 to $90 \%$ of patients, and strongly predict functional disability [5], unemployment [6], and quality of life [7]. Cognitive deficits are present since the early phase of the disease, in first episode schizophrenia [8] and are relatively stable during the course of the illness [9].

Cognitive rehabilitation seeks to improve cognitive functioning and real world functioning and has been divided into two main strategies, compensatory (e.g. cognitive remediation) and restorative approaches [10]. On, one hand, the goal of the restorative approaches are to improve or restore the impaired cognitive skill, through a range of repeated cognitive tasks, practice and strategy acquisition activities [11]. On the other hand, rather than aiming to improve cognitive functions per se, compensatory approaches bypass and compensate the functional impacts of cognitive deficits by engaging preserved cognitive functions (i.e. procedural memory) or by implementing environmental supports (i.e. alarm reminders, checklists) that sustain independent living and autonomy [12-14].

One of the crucial aspects of cognitive rehabilitation concerns the transfer of learned techniques and trained cognitive skills to real world functioning $[10,15]$. If there is large amount of evidence supporting the effects of cognitive remediation on improving cognitive functioning in schizophrenia, there is, however, poorer evidence of generalization to functional outcomes $[11,16]$. Individualized cognitive remediation and concrete objectives on people's everyday complaints may be an effective alternative to obtain a meaningfully lasting transfer of training to daily life [15]. Importantly, it has been suggested that compensatory approaches are generally more efficient than restorative ones and more easily generalized to daily activities [14].

Among the compensatory interventions that have been developed specifically for schizophrenia (Twamley et al., ), the Cognitive Adaptation Training (CAT) $[1,17]$ is the compensatory intervention that has received the most empirical support to date $[14,18]$. In this compensatory intervention, trained mental health specialists implement compensatory techniques such as environmental supports in the individual's environment to live more independently and achieve greater self-sufficiency. Several randomized controlled studies in chronic [1,14,19-21] and first episode psychosis $[18,22]$ have established that the CAT intervention improves multiple outcomes at follow-up such as orientation, hygiene, but also adherence to treatment, relapse, and quality of life with high session attendance rates [18].

However, implementing this type of intervention can be time-consuming, requiring trained practitioners and time to maintain CAT effects. As a consequence, cognitive rehabilitation is not widely available in 
community care and this may partly explain the large number of patients who remain dependent on family members for daily living activities [23].

In the same way, there is a large amount of evidence that integrating the family into care reduces family accommodation [24] and improves functional outcomes in individuals with schizophrenia $[25,26]$. However, to date, family interventions have mainly focused on expressed emotions (hostility, criticism or over involvement) to prevent relapses, re hospitalisations and reduce non compliance to treatment but no intervention focused on cognitive deficits [25].

To overcome these limitations, two groups of researchers [2,27] developed CAT for family, a manualized intervention who helps families throughout all different steps for achieving their relative's goals [27]. The manual guides the family and their loved one to build SMART (Specific, Measurable, Achievable, Realistic, and Timely) goals and to use compensatory tools to counter functional difficulties associated the disease encountered in daily living. Two small randomized studies showed improvements in community functioning and a significant decline of the caregiver-burden [2,27]. Consequently, these preliminary results indicate that raising families' awareness of the compensatory approach could reduce the caregiver's burden and reduce their loved one's difficulties with minimal professional help.

In addition to lacking qualified mental health practitioners able to provide cognitive remediation, (leading to long waiting times for initial assessment), another important barrier to mental health care is travel distance required to access care in a timely manner. One way to reduce this inequality is the emergence of remote care via telehealth. Among teleheath solutions, web-based psychological therapies and particularly Cognitive Behavior Therapy (CBT) seem promising and have the potential to increase access to evidence-based therapy and reduce the financial costs for institutions. A meta-analysis [28] found a small but significant effect size of self-guided iCBT compared to control conditions. Evidence supports the use of new technologies and particularly web-based interventions to a wide range of psychological problems such as depression [29], anxiety disorders [30] or insomnia [31].

It is therefore advisable to take a greater interest in web-based psychological interventions. Concerning individuals with schizophrenia, web-based interventions exist including psycho-education [32], CBT [33] psychosocial interventions [34] and CBT for auditory hallucinations [35]. Moreover, interventions are developing for family caregivers of elderly individuals to offset declines in their health and wellbeing [36].

\section{Gap and Research hypothesis}

Although, only one study using web-based cognitive remediation can be found in the scientific literature [37], this study demonstrates the effectiveness of cognitive remediation associated with a supported employment program to facilitate the return to work in a schizophrenic population .

However, up to date, there are no studies that include the family in a web-based cognitive remediation therapy. 
Therefore, this study aims to evaluate the effectiveness of a web-based cognitive remediation therapy which integrates a family member in the care of their loved one. Unlike other web-based therapy, we provide regular feedback with a therapist.

Indeed, the availability of webcam feedback with a specialized therapist shows better results than in the absence of feedback [38-40].

To date, we have not found in the literature any study assessing a cognitive remediation program with a compensatory approach carried out at a distance with the involvement of a family member.

Therefore, the objective of this study is to see whether a web-based cognitive remediation therapy with webcam feedback can prove to be effective in individuals with schizophrenia, and thus be proposed in areas that do not currently benefit from trained therapists.

\section{Originality and innovative aspects of the study}

This study is the first one to explore the efficacy of a web-based family cognitive adaptation training with webcam feedback. In this program, each participant receives a module of the Cognitive Adaptation Training each week and a 15-minute webcam feedback by a psychologist.

This type of intervention is expected to be developed in territorial areas where professionals are not trained to cognitive remediation. Therefore, this type of intervention may substantially lower the barriers to the deployment of cognitive and other psychosocial interventions for individuals with schizophrenia and their caregivers.

To our knowledge, this is a pioneering study on a cognitive remediation program with a compensatory approach carried out at distance with the involvement of the family.

\section{Objectives}

\section{Primary Objective}

The primary objective is to determine if schizophrenia patients and their families can benefit from a compensatory approach using webcam feedback versus a psycho-educational manual also with webcam feedback.

A total of 60 dyads consisting of one caregiver and one supported individual with schizophrenia is randomized to either a Web-based family Cognitive Adaptation Training or an Internet-based control condition. The primary outcome measured is the score on the life skills profile.

\section{Secondary Objective}

The secondary objectives are to assess change in the caregiver's burden, medication adherence and negative symptoms in the participants suffering from schizophrenia. This is examined over three time 
points (at inclusion - 3 months - 9 months), according to the study arms.

Other objectives are to evaluate at the 9 months follow-up the impact of the intervention on:

- Clinical symptoms: positive symptoms, depressive symptoms

- Major cognitive functions (memory and executive functions)

\section{Hypothesis}

The primary hypothesis is that:

1. Compared to the control condition (psycho-educational manual), family cognitive adaptation training will lead to better daily living functioning (post treatment and at 6 months follow-up).

The secondary hypothesis is that:

2. Compared to the control condition, family Cognitive Adaptation Training will lead to reduction of the family burden, the negative symptoms and a better quality of life for the patient and his/her family (post treatment and at 6 months follow-up).

\section{Methods: Participants, Interventions, And Outcomes}

\section{Participants}

60 individuals with schizophrenia and 60 family members will be recruited and will form 60 dyads. The study protocol has been approved by the Ethical Committee (CNRIPH: 19.03.19.49332) in accordance with the Helsinki Declaration.

Written informed consent will be obtained by all participants. The study report will comply with the Consolidated Standards of Reporting Trials (CONSORT) statement and CONSORT-NPT (nonpharmacological treatments) statement.

\section{Eligibility Criteria}

\section{Inclusion criteria for the patient group are:}

1) Age between 18 and 50 years old;

2) Patient who has given written informed consent to participate in the study, as well as written consent from their guardian for patients under guardianship, and/or from the trusted person for patients hospitalized in Third Party Request Psychiatric Care, Psychiatric Care at the request of a state representative and in a care program. If the patient is under curatorship, the curator will be informed of the study; 
3) Patients with a diagnosis of schizophrenia according to the Diagnostic and Statistical Manual of Mental Disorders 5 (DSM 5) after evaluation by the investigating psychiatrist during the preliminary medical examination;

4) Patients who can understand, speak and read French;

5) Obligation to be a member or beneficiary of a social security scheme.

\section{Inclusion criteria for the family member group are:}

1) Be under 75 years old for the family member group;

2) Having signed an informed consent form;

3) The family carer is actively involved in the care of his or her relative with schizophrenia assessed by having at least one contact per week with the patient (Kidd et al., 2016);

4) The carer has an internet connection with a video chat application;

5) Understand, read and speak French;

6) Obligation to be a member or beneficiary of a social security scheme

\section{Exclusion criteria for the patient group are:}

1) A history of severe head injury and/or neurological pathology with cognitive impact;

2) Patient who has benefited from individual or group cognitive remediation.

\section{Exclusion criteria for the Family caregiver group are:}

1) Diagnosis of dementia;

2) A history of neurological pathology (epilepsy, multiple sclerosis, parkinson's disease, amyotrophic lateral sclerosis, Huntington's disease).

\section{Study Design}

This study is a comparative, single blind randomized controlled, clinical trial designed to assess the efficacy of a Web-based family Cognitive Adaptation Training in individuals with schizophrenia.

Patients and family members meeting all of the inclusion criteria and none of the exclusion criteria are invited to participate in this study. After signing the consent form, they are randomized into one of the two interventions: Cognitive Adaptation Training or Psycho-education. 
Patients are assessed at baseline for cognitive functioning, life skills profile, symptomatology, quality of life and medication adherence. An assessment of the living space is also carried out. This assessment allows for the identification of missing supplies for daily living tasks and living conveniences, as well as the condition of the living space including housing safety, cleanliness and maintenance.

Participants are re-assessed at 16 weeks corresponding to the end of the intervention and 6 months later (with a delay of 15 days maximum) for cognitive functioning, life skills profile, symptomatology, quality of life, assessment of the living space and medication adherence. If the intervention is delayed, this intermediate assessment is performed as soon as the intervention is completed and within 15 days of the end of the intervention.

A clinical psychologist, blind to the randomization process, performs the assessments at 16 weeks and at 6 months. Another psychologist performs the first assessments and the therapy.

\section{Setting}

Patients are recruited from Professor Capdevielle's adult psychiatric unit of the University Hospital of Montpellier, France, which includes an expert center on schizophrenia (FondationFondaMental, Pr. Capdevielle), a day hospital, and a recovery center for people suffering from schizophrenia. Recruitment also takes place within family associations. The recruitment of patients is also done among the patients followed in Pr Capdevielle's department by the different doctors and psychologists; though a referral by a psychiatrist or psychologist is not a requirement to participate. Recruitment is for outpatients only.

This study protocol has been reported in accordance with the "SPIRIT 2013 Checklist: Recommended items to address in a clinical trial protocol and related documents" (Additional file 1).

\section{Justification of the study design}

This clinical trial is designed to assess the efficacy of a web-based cognitive remediation therapy including the family in patients with schizophrenia by comparing results from CIRCUS (Cognitive Remediation Program for Family Caregivers with Schizophrenia) intervention to a web-based psychoeducational program including family.

We choose a single blind study to be sure that the psychologist performing the evaluations at 16 weeks and 6 months is blinded to the randomization arm.

It is not possible for patients following Cognitive Adaptation Training to be in a blinding condition.

For the comparison group (active comparator), patients benefit from a web-based psycho-educational program that will allow us to compare two web-based interventions.

\section{Intervention description}


Comparative, prospective, randomized, study including two groups of (patient / caregiver) dyads:

- $\quad$ An intervention group consisting of a dyad (patient and family carer) benefiting from the webbased CAT intervention.

- A control group consisting of a dyad (patient and caregiver) with a psycho-educational manual (control group).

\section{Experimental Group}

Cognitive Adaptation Training for Caregivers:

- 15 sessions of the CAT intervention

- 1 session per week sent by e-mail

- A 15 minute video feedback with a psychologist (different to the psychologist who performs the last assessments)

- dyad: patient and a family member

The Cognitive Adaptation Training for Caregivers has been developed by Pr. Velligan and Pr. Kidd [2]. They created a manual accessible to people without any knowledge of cognitive deficits. This manual helps families to select specific cognitive-adaptative strategies with their relative to achieve targeted goals. This method was translated in French and divided into different modules. The CAT for caregivers' intervention is delivered according to a standardized treatment manual comprising of 14 modules addressing daily living activities over 15 sessions (Table 1 ). The manual includes psycho-education on common cognitive disorders in schizophrenia, setting a smart goal, and cognitive methods in different domains of daily living activities and problem solving.

One module is sent per week to the dyad (patient and family member) for reading and implementing daily. It is a collaborative discussion between the family member and the patient on how to implement these methods according to their goals. A week after receiving the manual, each dyad benefits from a 15 minute video feedback with a trained psychologist to identify the weekly goal, implement the weekly strategy and resolve any issues encountered by the dyad.

Table 1. Manual modules and therapeutic aims

\section{Modules Content}




\begin{tabular}{|ll|}
\hline 1 & Understanding the signs and symptoms of schizophrenia \\
\hline 2 & Executive functioning \\
\hline 4 & Developing a plan \\
\hline 5 & Basic strategies for everyday living: Overview, bathing and dressing \\
\hline 6 & Basic strategies for everyday living: dental hygiene, applying makeup and using the toilet \\
\hline 7 & Basic strategies for everyday living: Keeping the home clean and tidy \\
\hline 8 & Basic strategies for everyday living: Healthy eating and cooking \\
\hline 9 & Basic strategies for everyday living: Doing laundry and managing medication \\
\hline 10 & Strategies that go beyond the basics: Overview, grocery shopping and transportation \\
\hline 11 & Strategies that go beyond the basics: Managing money \\
\hline 12 & Strategies that go beyond the basics: Social skills, communication and leisure skills \\
\hline 13 & Strategies that go beyond the basics: Works skills \\
\hline 14 & Putting supports into practice \\
\hline
\end{tabular}

During the first session, the therapist gives feedback on the cognitive assessment, announces to the dyad the group they are in, and schedules the follow-up appointments. In order to improve adherence to treatment, appointments are reminded to the dyad by email one day before the scheduled appointment. Then, module 1 is sent to the dyad. A feedback of this module is done one week later. For each module, a visuo-consulting feedback is provided to the dyad with the psychologist. This feedback lasts between 15 and 20 minutes where the therapist can answer any technical questions about the environmental support, the place where it has been set up, motivational issues etc.

At the last feedback, the therapist makes sure to get feedback on the program and on the patient's progress.

\section{Control Group}

The control group receives a psycho-education manual, with a weekly reading module and a 15-minute webcam feedback by the psychologist.

- 15 sessions of the psycho-education manual

- 1 session per week sent by e-mail

- A 15-20 minutes video feedback with the psychologist (different to the psychologist who performs the last assessments) 
- dyad: patient and a family member

The psycho-educational manual was created within the unit by psychologists specialized in schizophrenia and behavioral and cognitive therapies.

The choice of the modules is based on factors that can improve healthy living, such as physical activity, nutrition and limited use of toxic substances.

The interactive manual has been created to allow the formulation of SMART goals and the implementation of new behaviors to enhance improvement.

\section{Modules Contents}

\begin{tabular}{|ll|}
\hline 1 & Physical activity \\
\hline 2 & Physical activity part 2 \\
\hline 3 & Eating habits \\
\hline 5 & Eating habits part 2 \\
\hline 6 & Smokration \\
\hline 7 & Smoking part 2 \\
\hline 8 & Alcohol and drugs \\
\hline 9 & Alcohol and drugs part 2 \\
\hline 10 & Stress and relaxation \\
\hline 11 & Sleep \\
\hline 12 & Sleep part 2 \\
\hline 13 & Respecting the drug treatment \\
\hline 14 & Social relationship \\
\hline 15 & Social relationship part 2 \\
\hline
\end{tabular}

\section{Outcomes measurements and other data collected}

\section{Primary outcome measures}

1. Life Skills Profile Score (LSP-39) [ Time Frame: Baseline-16 weeks - 6 months ] 
The "Life Scale Profile" (LSP), validated in French by Mohr et al [41] is a scale used to assess the subject's life skills profile, particularly for patients with schizophrenia. It is a self-administered questionnaire based on the patient's functioning during the last week (excluding any acute or relapse phase). The scale is composed of 20 items. The instruction is to rate each item based on a Likert scale from 1 to 4: "always, usually, rarely and never". The lower the score, the more the patient has a deficit profile in terms of life. The maximum score is 80 . The LSP scale assesses 5 dimensions of life skills: sociability, selfmanagement, treatment compliance, antisocial behaviors and strange behaviors. The total duration of the test is about 30 minutes.

\section{Secondary outcome measures}

1. Global score of the Zarit burden interview [ Time Frame: Baseline-16 weeks - 6 months ]

Zarit's burden scale $[42,43]$ is a 22 -item self-questionnaire measuring the burden experienced by a caregiver of an individual with a chronic disorder. The total score, which is the sum of the scores obtained from the 22 items, varies from 0 to 88; a score less than or equal to 20 indicates a low or zero load; a score between 21 and 40 indicates a low load; a score between 41 and 60 indicates a moderate load; and a score greater than 60 indicates a high load.

2. Patient quality of life: S-QoL 18 [ Time Frame: Baseline-16 weeks - 6 months ]

The quality of life of patients is assessed using a French scale, the Quality of Life Scale (S-QoL 18). The S-Qol 18 is a shortened version of the S-QoL 41 [44] scale whose validity and psychometric properties have been validated in patients with schizophrenia [45]. This self-administered questionnaire assesses the impact of patients' difficulties on their quality of life through 8 dimensions: psychological well-being, self-esteem, family relationships, friendly relationships, resilience, physical well-being, autonomy and emotional life and a total score (index score). The duration of the test is 7 minutes. Dimension and index scores range from 0 , indicating the lowest quality of life, to 100 , the highest quality of life.

All participants (patients with schizophrenia and their family caregiver) complete this questionnaire

\section{Caregiver quality of life: S-QoL 18 [ Time Frame: Baseline-16 weeks - 6 months ]}

The quality of life of caregivers is assessed by the Quality of Life Scale: As for patients, we will use the Quality of Life Scale (S-QoL 18). The self-administered questionnaire assesses the impact of patients' difficulties on their quality of life through 8 dimensions: psychological well-being, self-esteem, family relationships, friendly relationships, resilience, physical well-being, autonomy and emotional life. The duration of the test is 7 minutes. Dimension and index scores range from 0 , indicating the lowest quality of life, to 100 , the highest quality of life.

4. Negative symptoms [ Time Frame: Baseline-16 weeks - 6 months ] 
Clinical Assessment Interview for Negative symptoms (CAINS) [46]. CAINS is a recently developed tool to evaluate the two dimensions of negative symptomatology highlighted in the literature, i.e. the motivation dimension and the decreased emotional expressivity dimension. Factor analysis of the CAINS showed a 2-factor structure, a motivation-pleasure (MAP) factor consisting of 9 items (items 1-9) and an expression (EXP) factor consisting of the remaining 4 items $[46,47]$. The motivation-pleasure dimension score range from 0 to 36 and the expression dimension score range from 0 to 16 . A composite of the two dimensions can be computed. Total scores may vary between 0 and 52, a higher score implies that the negative symptoms are significant and that they are responsible for impaired functioning.

5. Patient medication adherence [ Time Frame: Baseline-16 weeks - 6 months ]

Medication Adherence Rating Scale [48]. This scale is one of the most widely used tools to assess compliance in individuals with schizophrenia. This is a self-administered questionnaire with $10 \mathrm{yes} / \mathrm{no}$ items offering a total score from 0 (low compliance) to 10 (high compliance).

6. Depressive symptoms [ Time Frame: Baseline-16 weeks - 6 months ]

Calgary Depression Scale for Schizophrenia (CDSS) [49] translated in French [50] is a nine item clinician rated outcome measure that assesses the level of depression in people with schizophrenia. It distinguishes depressive symptoms from negative symptoms. Scores range from 0 to 27 , a higher score indicating increased level of depression.

\section{Cognitive Functioning}

1. Premorbid Intellectual Quotient (IQ): fNART [Time Frame: Baseline- 6 months]

The premorbid IQ is assessed by the French National Reading Test (fNART) [51]. Based on the ability to pronounce 40 irregularly spelled words (one point per correct answer). This test provides an estimate of the participant's premorbid IQ. Scores range from 0 to 40.

2. Episodic Memory: California Verbal Learning Test (CVLT) [Time Frame: Baseline- 6 months]

The CVLT [52] was developed to evaluate in a multifactorial way verbal learning and memory. It has the particularity to quantify several cognitive components of verbal memory in only one test. Its duration is around 20 minutes. A list of 16 words (four from each of four semantic groups) is presented orally to the participants, who are required to recall the words immediately, after short, and long delays, and also with and without semantic cues. A recognition task is then administered. The test includes the following measures to reflect learning and recall, also called primary variables: Total Trial 1-5, Short Delay Free Recall (SDFR), Short Delay Cued Recall (SDCR), Long Delay Free Recall (LDFR), and Long Delay Cued Recall (LDCR). The CVLT also includes other variables that reflect processing of information and other cognitive processes such as Semantic Clustering and Serial Clustering, Region effects, Intrusions, and Perseverations. 
3. Working Memory: Letter Number sequence - Wechsler Adult Intelligence Scale IV (WAIS IV) [Time Frame: Baseline- 6 months ]

Working memory is assessed using the WAIS IV letter-number sequence test [53] which is a validated measure of working memory. The purpose of the task is to locate the sequence orally by ordering the numbers in ascending order and the letters in alphabetical order. The test measures the subject's ability to retain, in the short-term information, while manipulating it mentally.

\section{Evaluation specific for the patient, in order to determine the type of compensatory and environmental intervention:}

1. Wisconsin Card Sorting Test (WCST) [Time Frame: Baseline- 6 months]

The WCST [54] is a test to study mental flexibility and abstraction skills. The subject is presented with 4 cards that differ in color of the shape of the items on each card (circles, squares, triangles, etc.) and the number of items on each card. The task is to categorize the cards. The participant is instructed to organize the cards as they wish (by color, shape or number of shapes). The examiner gives feedback ('yes' or 'no') if the chosen criterion is the right one. The participant has to adapt his behavior when the examiner decides to change the criterion. The dependent variables are: the number of well-placed cards (score ranges from 0 to 128), the number of categories achieved (score ranges from 0 to 6 ), the number and percentage of errors, the number and percentage of persevering errors.

2. Test of Verbal Fluency [Time Frame: Baseline- 6 months]

The test of Verbal Fluency [55] evaluates access to lexical stock as well as certain executive processes such as mental flexibility, verbal inhibition and updating in working memory. This test includes a semantic part and a lexical part. The participant in each part is asked to produce the most words in 2 minutes. In the lexical part the participant is asked to give the most words in two minutes starting with a given letter $(P)$. In the semantic part, the participant is asked to name the largest number of words belonging to an animal category in two minutes. The dependent variables are the total number of words for each condition, the number of repetitions, and the number of errors.

3. Evaluation of behavior related to frontal functions (FrSBe) [Time Frame: Baseline- 6 months]

The Frontal Systems Behavior Scale (FrSBe) [56] comprising of 46 items, evaluates three types of behaviors associated with executive/frontal dysfunction: apathetic, disinhibited and dysexecutive behaviors. This scale is completed by the caregiver on his or her loved one suffering from schizophrenia. Three sub-scores (apathetic, disinhibited, dysexecutive) constitute the dependent variables and are obtained by summing each type of item respectively.

4. Evaluation of the CAT techniques put in place [Time Frame: each week] 
An evaluation of the degree of implementation of the techniques taught in the CAT for family is made by noting both the frequency and type of CAT strategies used via a semi-structured interview [2] at each session. Two variables are noted: number of techniques used and frequency of use of each technique.

\section{Participant Timeline}

\section{Planned Trial Calendar}

Planned regulatory period: 3 months

Planned duration of recruitment: 18 months

Duration of participation of each subject: 10 months

Planned statistical analysis/valorization period: 3-6 months

Planned total trial period: 36 months

\section{Visit schedule}




\begin{tabular}{|c|c|c|c|c|}
\hline Item & Screening & Baseline & & Final Visit \\
\hline & V0 & V 1 & V 2 & V 3 \\
\hline Date & & $\begin{array}{l}\text { Day } 0+15 \\
\text { days }\end{array}$ & $\begin{array}{l}4 \text { months } \\
\pm 15 \text { days }\end{array}$ & $\begin{array}{l}6 \text { months } \\
\pm 15 \text { days }\end{array}$ \\
\hline Informed consent & $\mathrm{x}$ & & & \\
\hline Medical history & & $x$ & $x$ & $x$ \\
\hline Treatment & & $x$ & $x$ & $x$ \\
\hline Socio-demographic data & & $x$ & $x$ & $x$ \\
\hline \multicolumn{5}{|l|}{ PATIENT } \\
\hline \multicolumn{5}{|l|}{ Clinical evaluation } \\
\hline PANSS & & $x$ & $x$ & $x$ \\
\hline CAINS & & $x$ & $x$ & $x$ \\
\hline \multicolumn{5}{|l|}{ Functional evaluation } \\
\hline CDSS & & $x$ & $x$ & $x$ \\
\hline MARS & & $x$ & $x$ & $\mathrm{x}$ \\
\hline LSP & & $x$ & $x$ & $\mathrm{x}$ \\
\hline SQOL-18 & $x$ & $x$ & $x$ & $x$ \\
\hline \multicolumn{5}{|l|}{ Cognitive assessment } \\
\hline fNART & & $x$ & & $x$ \\
\hline CVLT & & $x$ & & $x$ \\
\hline $\begin{array}{l}\text { WAIS IV (letter-number } \\
\text { sequence) }\end{array}$ & & $x$ & & $x$ \\
\hline WCST & & $x$ & & $x$ \\
\hline Verbal fluency & & $x$ & & $x$ \\
\hline Environmental assessment & & $x$ & $x$ & $x$ \\
\hline CAREGIVER & & $x$ & $x$ & $x$ \\
\hline SQOL-18 & & $x$ & $x$ & $x$ \\
\hline ZARIT & & $x$ & $x$ & $x$ \\
\hline $\mathrm{FrSBe}$ & & $x$ & $x$ & $x$ \\
\hline
\end{tabular}

Determination of sample size and power calculation 
Professionally conducted cognitive remediation programs have an effect size of 0.7 [11]. In Kidd [22], where the program was family led, the observed effect sizes ranged from 0.6 to 1.09 . We therefore hypothesize an effect size of 0.7 in our study. In our usual clinical practice, caregivers of patients suffering from schizophrenia are highly adherent to care. By recruiting 40 dyads per group, with 2 dyads lost to follow-up, we will be able to show an effect size of 0.75 , with a power of $90 \%$, at $5 \%$ alpha risk. The investigators contacted psychiatrists on the different psychiatric units of the hospital to present the research project. The investigators also made presentations at family association meetings and contacted private psychiatrists. Flyers were also distributed at each presentation.

\section{Methods: Assignment Of Interventions}

\section{Randomization/matching}

After checking for all inclusion and non-inclusion criteria by the study investigator, patients are randomized to either of the study interventions (Cognitive Adaptation Training or Psycho-education). Allocation concealment will be guaranteed as the randomization will not be released until the participant has been recruited into the trial, taking place after the baseline measurements have been done.

Randomization is performed by the Clinical Research Unit of the Department of Medical Information of the Montpellier University Hospital. Randomization is accessible online and is programmed using the data Capture Software System (EnnovClinical randomization module) according to a minimization algorithm with a 1:1 ratio. The trial manager will be responsible for setting up and testing the randomization process, and assuring that study groups of approximately the same size are produced.

The patient number will be automatically generated by EnnovClinical software.

\section{Blinding}

The psychologist performing the inclusion and the therapy is not the same psychologist performing the 16 weeks and 6 month assessments. This psychologist is blinded to which intervention participants have been allocated to, the Cognitive Adaptation Training or psycho-educational program. Patients and the psychologist performing interventions are asked to avoid telling the assessor to which group they belong to. The data analyst that is performing the analysis at the conclusion of the trial is also blinded as to what group the data corresponds to. Unblinding should occur only in exceptional circumstances in case of any adverse events (AE) which are reported immediately to the investigator.

Assessment of adherence: None

Concomitant care: patients cannot be included in another protocol or benefit from another cognitive remediation therapy. This is specified on the consent form.

\section{Methods: Data Collection, Management, And Analysis}




\section{Data collection}

Data are collected or reported in a Case Report Form (CRF). Once a participant has been enrolled and randomized into their study arm, the study site puts in place every effort to follow the participant throughout the course of the study. In order to improve adherence and prevent loss to follow-up, we organize phone calls and/or emails that are sent before the intervention to remind patients of their appointments.

Some patients are following day hospital care, which we believe increase adherence to the study.

Furthermore, each evaluation is planned in accordance with each patients' schedule and all patients are phoned 24 hours before their evaluation to remind them of their appointment.

If a patient misses their appointment, the psychologist calls him/her. All attempts are documented in the patient's medical records. In case of discontinuation, the investigator performs all examinations scheduled for the final study visit, which includes recording of any AEs.

In any case, the patient is treated in accordance with standard care in the center.

Investigators should make every effort to minimize the number of patients lost to follow-up and to obtain a maximum of information on patients lost to follow-up, particularly in the search for any AEs.

\section{Data management and monitoring}

The patients are identified by the patient code:

- Number of the patient in the center by chronological order of selection (2 digits)

- First letter of first name

- First letter of family name

Source documents

Source documents are original documents and patient records from which patient data are reported in the CRF. The investigator must commit to allow direct access to data sources in the study during inspections or audits.

The source documents that may be used to complete the CRF are the patient records. The investigator commits to allow direct access to the patient records during inspections or audits.

The encrypted data is transmitted to the data-management center via a secure internet connection.

The CRF is designed to capture all relevant medical information from patients included in the project. The study methodologist has a restricted reader-only access to all data in order to monitor the progress and 
the quality of the data.

The purpose of an audit is to confirm that the study is conducted as per protocol, International Conference on Harmonisation-Good Practice (ICH-GCP) and applicable regulatory requirements, that the well-being and the rights of the subjects enrolled have been protected, and the data relevant for the evaluation of the investigational product have been recorded, processed, and reported in compliance with the planned arrangements. The investigators permit a direct access to all study documents, drug accountability records, medical records and source data.

\section{Data processing}

Individual data needed for the study analysis must:

- Be entered in the CRF as they are obtained, for both clinical and paraclinical data

- Be anonymized by the investigator

- Be authentified by an electronic signature of the investigator

- All be entered, and missing data must be justified

\section{Site training and monitoring procedures}

The monitor must present the protocol and all procedures related to the study during an initiation visit performed before the first patient is included. A case report form of completion guidelines is provided to the Investigator.

The monitor is allowed to have access to all source documents needed to verify the entries on the CRF and other protocol-related documents.

To ensure accurate, complete, and reliable data, the sponsor or its representatives do the following:

- Provide instructional material to the study sites, as appropriate

- Provide a start-up training session to instruct the investigator(s) and study coordinator(s). This session give instruction on the protocol, the prompt and full completion of the clinical report forms, study procedures, and the transmission of data in a timely manner to the clinical database for statistical analyses.

- Make periodic visits to the study site

- Be available for consultation and stay in contact with the study site personnel by mail, telephone, and/or fax

- Review and evaluate Case Report Form data and use 
- Conduct quality review of database

Routine monitoring visits are made by the monitors, designated by the sponsor to check compliance with the protocol, the completeness, accuracy and consistency of the data, and adherence to good clinical practice (GCP).

The principal investigator must ensure that CRFs are completed in a timely manner and must allow periodical access to CRFs, patient records, drug logs and all other study-related documents and materials.

The investigator agrees to provide the monitor direct access to the subjects' source data, which exist in the form of hospital records and patient files and notes.

Considering the low risks in this study, there is no formal Data Safety and Monitoring Board for this study.

\section{Trial governance and monitoring}

The sponsor is responsible for the administrative and financial management of the study, for its quality, and for the choice of the investigators and sites. The sponsor is also responsible in the assessment of $\mathrm{SAE}$, in reporting any suspected unexpected serious adverse reaction (SUSAR) and new events according to the legislation and good clinical practices, to the competent authorities. In addition to the expedited reporting, the sponsor shall submit, once a year, throughout the clinical trial or on request, a safety report to the competent authorities and the Ethics Committee (EC) of the concerned Member States taking into account all new available safety information received during the reporting period. The aim of the annual safety report is to assess the safety conditions of subjects included in the concerned trial(s). It should be the same for the competent authorities (CA) concerned and the Ethics Committee concerned. The sponsor should promptly notify all concerned investigators and the regulatory authorities of findings that could affect adversely the safety of subjects, impact the conduct of the trial, or alter the CA approval/EC's favorable opinion to continue the trial.

Investigators are responsible for the study quality, the respect of the GCP and protocol during the study. The investigators ensure information on the study is given to participants and checks that they have given their informed consent. Investigators are also responsible in contacting an appropriate pool of potential subjects and inviting them to participate in the study as well as ensuring the participants' eligibility. A core project management team consisting of the principal investigator, all co-investigators meet monthly to oversee the general running of the trial.

Psychologists are in charge of assisting the investigators for the following:

- Management of the study on site

- Reporting of data in the CRF

- Verification of respect of study procedures 
- Recruitment

- Deliver the CIRCUS intervention

The investigator is responsible in presenting the study (information, consent, inclusion) and following the patients during the entire study.

The methodologist is in charge of the quality of the data and the statistics.

GCP requires that the clinical protocol, any protocol amendments, the informed consent and all other forms of patient information related to the study (e.g. advertisements used to recruit patients) and any other necessary documents be reviewed by an Independent Ethics Committee (IEC)/Institutional Review Board (IRD). IEC/IRB approval of the protocol, informed consent and patient information and/or advertising, as relevant, is obtained prior to the authorization of enrolling patients in the study. The study shall be conducted as described in this approved protocol. Any revisions/amendments to the protocol are not permitted without prior approval by the study steering committee. Any amendments to the protocol require IEC/IRB approval prior to the implementation of any changes made to the study design.

\section{- Statistical Analysis}

The statistical analysis plan (SAP), covering all the analyses to be performed on all data, will be written before database lock for final extraction.

For all collected variables, descriptive statistics will be shown for both study arms in order to verify the initial comparability of the groups. For metrics variables, a check as to whether the data can be assumed to be normally distributed will be calculated, whereas for skewed variables, means and ranges will be given. For categorical variables group proportions and contingency tables will be prepared.

All tests of efficacy will be conducted on an intention to treat (ITT) basis using, in case of missing data, the Last Observation Carried Forward method, and/or multiple or mean imputation. A per protocol sensitivity analysis will also be performed taking into account only patients who completed the whole study without any major protocol deviations. This will enable us to reinforce the stability of results obtained by the ITT approach.

Significance level will be set at $p<0.05$. All statistical analyses will be performed using SAS software (SAS Institute, Cary, NC, USA).

\section{Main endpoint analyses}

The efficacy of the CIRCUS intervention will be studied by comparing the change in subject's life skills profile (using the score on the LSP questionnaire) between inclusion/randomization and the 9month follow-up in the intervention group compared to the psycho-educational group. 
For each subject, the relative difference (delta) between the inclusion and the 9-month life skill profile will be calculated. The normality of the delta score will be tested using the Shapiro Wilk's test. If the distribution is skewed, a transformation will be performed to normalize it.

Study arms will be compared between inclusion scores and the 9-months scores using mean differences. These comparisons will be done using Student's T-test and general linear regression models adjusting for covariates. Socio-demographic and clinical variables will be tested as potential confounders using general linear regression models, with the p-value set at 0.10 .

A second analysis will be carried out with the general life skills profile as the outcome variable, adjusting for general life skills profile score at inclusion.

\section{Secondary endpoint analysis}

The secondary outcome analyses will be assessed similarly to the main endpoint analyses. Specifically, for normally distributed variables, means and standard deviations will be used to conduct the analyses, whereas for skewed variables, medians and ranges will be used.

- A secondary outcome variable will be constructed, measuring the change in life skills profile (based on LSP questionnaire) between inclusion scores and the 3-months follow-up, representing the end of the CIRCUS intervention (for both study arms). Change in life skills profile over the three time points using mean differences in scores between the intervention and the psycho-educational arms, will be compared using a mixed-model, taking into account the repeated and reversible time-dependent outcome measures.

- Assessment of the effect of the intervention on a range of exploratory outcomes (clinical and cognitive factors) measured at the 9-months follow-up will be carried out using either linear regression models or logistic regression models, depending on the type of variable (continuous or binary). These scores will be compared to the initial scores obtained at inclusion. For each outcome variable, the model will be adjusted for the baseline value. These secondary analyses will include measures of:

- General symptomatology -PANSS scores

- Severity in negative symptoms -CAINS scores

- Depression-CDSS scores

- Quality of life -S-QoL scores for patient and caregiver

- Patient compliance-MARS scores

- Burden experienced by a caregiver -Zarit scores

- Executive frontal dysfunction -FrSBe scores 
- Intellectual ability -FNART scores

- Learning and recall -CVLT scores

- Working memory - Letter number sequence of the WAIS IV scores

- Mental flexibility and abstraction skills - WCST scores

- Lexical stock and verbal inhibition -Verbal fluency scores

- Additionally, a cross-sectional analysis of baseline data will be performed to study the psychological and cognitive factors associated with low life skills profile using general linear regression models.

Modifications to the initial SAP will require an amendment to the protocol. Exploratory statistical analysis not documented in the initial SAP will be discussed by the co-investigators and statisticians, in order to establish their importance and acceptability with respect to the study's main and secondary objectives.

\section{Discussion}

Cognitive deficits are highly prevalent in schizophrenia and have an impact on people's quality of life and autonomy. Given the significant impact of cognitive deficits on daily living in individuals with schizophrenia, studies have begun to explore methods to remediate cognitive impairments within this population. Specifically, cognitive remediation and especially the compensatory approach is designed to improve an individual's abilities through the introduction of compensatory and/or adaptive strategies.

Compensatory approaches have been demonstrated to improve functioning in people with schizophrenia. However, only a minority of patients can benefit from the compensatory approach due to lack of access to trained professionals in remote areas.

For this reason, the compensatory approach has also been adapted to guide families and their loved ones to achieve their goal without the intervention of a professional at home. The CAT for family has demonstrated its effectiveness.

Similarly, in order to reach more people, developing web-based therapies seems judicious. Indeed, webbased cognitive remediation has shown its efficiency, but it has never included a family member in the loved one's therapy.

This randomized controlled study aims to identify the efficiency of a web-based cognitive remediation intervention including family with the use of video feedback.

This is a novel approach and may increase dissemination of cognitive remediation of compensatory approach in all areas. Cognitive deficits are an important factor in the disease and have a major impact on different areas of life. By setting SMART goals and compensating for these deficits, we hope to improve the quality of life and global functioning within this population. This approach is part of a recovery process and may change the common view of schizophrenia. 
Similarly, it is important to develop web-based therapies at a time when we are dealing with COVID-19 and the health measures taken to prevent the spread of the pandemic.

From this perspective, the emergence of web-based therapy enables continuity of care and ensures the link with our patients.

\section{Limitations:}

One of the first limitations of this project may be the difficulty to maintain feedback via webcam every week. It is sometimes difficult for participant/carer dyads to make themselves available. As a result, some follow-ups may last longer than the initial sixteen weeks.

However, the scientific literature shows that web-based therapies can become less effective due to a decrease in the level of commitment of the participants. Studies have shown that regular contact (via email or visual contact) helps to maintain commitment. Similarly, the feeling of control of the participants, the promotion of autonomy, as well as the feeling of being concerned about the subject are important factors in adherence to web-based therapies [34].

\section{Abbreviations}

- CAINS: Clinical Assessment Interview for Negative Symptoms

- CAT: cognitive adaptation training

- CIRCUS: Cognitive Remediation Program for Family Caregivers in Schizophrenia

- CDSS: Calgary Depression Scale for Schizophrenia

- CONSORT: consolidated standards of reporting trials;

- CVLT: California Verbal Learning Test

- DSM-5: Diagnostic and Statistical Manual of Mental Disorders, fifth edition;

- EECFF: Echelle d'Evaluation Comportementale lié aux Fonctions Frontales

- fNART: French National Adult Reading Test

- GCP: Good Clinical Practice

- ICBT: Internet-based Cognitive and Behavioral Therapy

- IEC/IRB: Independent Ethics Committee/ Institutional Review Board

- IQ: Intellectual Quotient

- LSP: Life skills profile

- MARS: Medical Adherence Rating Scale

- NPT: non-pharmacological treatments

- SMART: specific, measurable, achievable, realistic, timely

- S-QoL: Quality of Life Scale 
- SUSAR: suspected unexpected serious adverse reaction;

- WAIS IV: Wechsler Adult Intelligence Scale IV

- WCST: Wisconsin Card Sorting Test

\section{Declarations}

\section{Ethical considerations and confidentiality}

There are to our knowledge no potential risks involved with participating in the intervention proposed in this study other than the usual risk linked to moderate walking.

Risks concerning neuropsychological and clinical evaluations are weak: anxiety, tension, tiredness.

Regarding the vigilance of the project, which are responsibilities of the investigator and sponsor, the reporting of serious adverse events (SAE) and annual safety reports will be monitored and carried out in accordance with regulations

\section{Collection of AES}

AEs will be collected non-systematically through spontaneous report by participants or other members of the team. Complete and appropriate data on all AEs experienced during the clinical trial will be recorded on the $A E$ form of the CRF on an ongoing basis for the duration of the study. Each AE report shall include a description of the event, an assessment of its seriousness, its duration, intensity, relationship to the study medication, other causality factors (if any), any concomitant medication dispensed, actions taken with the study drug or other therapeutic interventions, and outcome at the end of the observation period. For each $A E$, a separate $A E$ form will be filled out.

All AEs (whether expected or unexpected) will be recorded in the CRF and will be reported in the subsequent trial publications except for the following:

Admission for social or administrative reasons,

- Hospitalization predefined by the protocol,

- Hospitalization for scheduled medical or surgical treatment prior to research

AEs will be followed up until their resolution or stabilization. However, the observation period will be cut off after the last patient has finished his final visit (LPLV).

\section{Notification of SAES}

All SAEs occurring during the study must be reported on the SAE form to the Pharmacovigilance Clinical Trial Department of CHU Montpellier handling pharmacovigilance matters in this study on behalf of the sponsor. 
The Pharmacovigilance Clinical Trial Department of the sponsor must be informed immediately (i.e. within 24 hours) of the occurrence of any SAE by fax or by e-mail.

The investigator is required to include any investigations as may be indicated to elucidate the nature or the causality of the SAE. This may include additional lab tests, histopathological examinations, and consultations with other healthcare professionals or if the patient dies, any post mortem findings. The relation between the event and the study should be evaluated by the investigator.

After the initial report, the investigator is required to follow each patient until the end of the SAE and to provide further information on the patient's condition to the sponsor. The investigator must also record all SAEs in the CRF.

Some serious adverse events that do not require immediate reporting, will only be documented on the $\mathrm{CRF} /$ source data such as:

- Serious adverse events occurring after given informed consents, but before starting study procedures/application of study medication;

- Hospitalizations

\section{Trial withdrawal or discontinuation}

Study participation is voluntary. The subjects have the right to withdraw their consent from the study at any time and for any reason, without jeopardizing their medical care. The investigator also has the right to withdraw patients in agreement with the sponsor or his delegate. The investigator also has the right to withdraw subjects from the trial in the event of concurrent illness, adverse events, and treatment failure after a prescribed procedure, protocol violations, and other reasons.

Subjects must be withdrawn from the study under the following circumstances:

- The subject withdraws consent

- Development of another illness or condition which would interfere with the subject's continued participation

- Discovery that the subject entered the clinical trial in violation of the protocol or occurrence of a significant protocol violation during the clinical trial

- Any situation that in the opinion of the investigator would pose inacceptable risks to the subject if trial participation is continued

For any discontinuation the investigator should obtain all required details and should document the date and reason of the premature termination in the CRF.

If the reason for withdrawal is an $A E$, the specific event will be recorded in the CRF. 
The investigator will make thorough efforts to document the outcome.

Patients prematurely withdrawn or lost to follow-up will be analyzed at least in the safety set and in the conditions of confidentiality.

\section{Definition of the End of Trial}

The end of the study is the date of the last visit of the last subject undergoing the intervention.

\section{Stopping rules}

The sponsor may discontinue the study at any time for any of the following reasons:

- Insufficient rate of recruitment

- Serious unsolvable problems with data quality

- Occurrence of unpredictable conditions at the trial site demanding the discontinuation of the clinical trial

- Upcoming scientific findings or ethical concerns during the conduct of the clinical trial demanding the discontinuation

The reasons for discontinuation of the clinical trial should be precisely documented.

If an investigator has ethical concerns about the conduct of the clinical trial the coordinating investigator has to be informed without delay.

In case of premature discontinuation of the clinical trial by the sponsor or the coordinating investigator, the responsible national competent authorities and ethic committees must be informed within the timelines required by European and national regulations.

The Competent Authorities may suspend or prohibit a study if it considers that the conditions of authorization are not being met or has doubt about the safety or scientific validity of the study. In case of a SUSAR, the investigator will inform the sponsor for stopping the trial and for informing regulatory agencies.

Other than the annual safety evaluations, mentioned above, we do not plan to carry out any interim analyses.

\section{Ethics Approval and Consent to participate}

This study was approved by the French ethics committee (CPP Sud Est VI, Montpellier, France) in February 2019 (CNRIPH: 19.03.19.49332) and study initiation is currently ongoing. Informed consent will be obtained from all study participants before any study procedures are performed. In accordance with GCP, prior to collecting the consent form or the non-opposition form, the subject or subject's legal 
representative will be informed by appropriate study personnel about all aspects of the trial that are relevant to making the decision to participate, and will have sufficient time and opportunity to ask any questions. Subjects will be clearly and fully informed about the purpose, potential risks, and other critical issues regarding the clinical studies in which they have volunteered to participate.

Documentation of the discussion will be recorded in the subject's medical record. Documentation of the consent process will be recorded in the source documents.

The subject will be informed that he/she should notify the investigator of any other medical measures during the study period and that he/she cannot simultaneously take part in another study without informing the investigator.

Subjects must also be informed that participation is voluntary and that they may withdraw from the study at any time, without prejudice to their current or future care. Documentation of the discussion and the date of informed consent must be recorded in the subjects' medical record.

Up to this date, there are no additional studies planned using the participants' data. Any additional studies using the participants' data will require a new clinical protocol and will be submitted to the IEC/IRB for approval.

\section{Trials Status}

At the time of manuscript submission, recruitment has launched and participants are currently being enrolled in the study. The estimated completion date for primary data collection is June 2022.

The protocol published here is version 1 dated February 21th 2019.

\section{Sponsorship}

The trial sponsor is the University Hospital of Montpellier,

Centre Administratif André Bénech;

191 Avenue du Doyen Gaston Giraud, 34295 Montpellier

Legal representative of sponsor: Thomas Ludec

General director - CHU of Montpellier

dg.secretariat@chu-montpellier.fr

In collaboration with the National Institute of Health and Medical Research, France (Institut National de la Santé et de la Recherche Médicale, France).

\section{Publication policy}


Any oral or written communication of research must receive the prior agreement of the coordinator investigator and sponsor. The $\mathrm{CHU}$ of Montpellier is holder of data and no use or transmission to a third party can be done without its prior agreement. The $\mathrm{CHU}$ of Montpellier must be referenced as research sponsor. The study results will be given to the participating members of the study and to the patients upon request.

Ethics approval and consent to participate: All experimental methods will be carried out in accordance with the ethical guidelines determined by the National Ministry of Health, Labour and Welfare and the Declaration of Helsinki. The Ethical Committee has approved the study protocol (CNRIPH: 19.03.19.49332).

Consent for publications: Institutionals consents forms were used and are available on request.

Availability of data and materials: The datasets generated and/or analysed during the current study are not publicly available due to participant confidentiality reasons but are available from the corresponding author on reasonable request.

Competing Interests: The authors declare that they have no competing interests.

Funding: The study was financed by a French National University Hospital Clinical Research Grant, Montpellier, 2019, (\#UF7708). The funder is not involved in the design of the study, manuscript writing or collection of data, and the funder will not be involved in the data analysis or interpretation and manuscript writing in the future.

Authors' contributions: SR, DC and MA conceived the study. DC, SR, and MA designed the trial. SR, AG and TD designed the cognitive remediation therapy. SR, DC, MA, YL and NR contributed to the development of the trial protocol. $C D$ is the trial statistician. All authors read and approved the final manuscript.

Acknowledgements: Not applicable

\section{References}

1. Velligan DI, Bow-Thomas CC, Huntzinger C, Ritch J, Ledbetter N, Prihoda TJ, et al. Randomized Controlled Trial of the Use of Compensatory Strategies to Enhance Adaptive Functioning in Outpatients With Schizophrenia. Am J Psychiatry. août 2000;157(8):1317-28.

2. Kidd SA, Kerman N, Ernest D, Maples N, Arthur C, de Souza S, et al. A pilot study of a family cognitive adaptation training guide for individuals with schizophrenia. Psychiatr Rehabil J. juin 2018;41(2):109-17.

3. Fioravanti M, Carlone O, Vitale B, Cinti ME, Clare L. A Meta-Analysis of Cognitive Deficits in Adults with a Diagnosis of Schizophrenia. Neuropsychol Rev. juin 2005;15(2):73-95. 
4. Fioravanti M, Bianchi V, Cinti ME. Cognitive deficits in schizophrenia: an updated metanalysis of the scientific evidence. BMC Psychiatry. déc 2012;12(1):64.

5. Green MF, Horan WP, Lee J. Social cognition in schizophrenia. Nat Rev Neurosci. oct 2015;16(10):620-31.

6. Evensen S, Wisløff T, Lystad JU, Bull H, Ueland T, Falkum E. Prevalence, Employment Rate, and Cost of Schizophrenia in a High-Income Welfare Society: A Population-Based Study Using Comprehensive Health and Welfare Registers. Schizophr Bull. mars 2016;42(2):476-83.

7. Eack SM, Newhill CE. Psychiatric Symptoms and Quality of Life in Schizophrenia: A Meta-Analysis. Schizophr Bull. 17 juill 2007;33(5):1225-37.

8. Bora E, Murray RM. Meta-analysis of Cognitive Deficits in Ultra-high Risk to Psychosis and FirstEpisode Psychosis: Do the Cognitive Deficits Progress Over, or After, the Onset of Psychosis? Schizophr Bull. 1 juill 2014;40(4):744-55.

9. Bowie CR, Harvey PD. Cognitive deficits and functional outcome in schizophrenia. Neuropsychiatr Dis Treat. déc 2006;2(4):531-6.

10. Bowie CR, Bell MD, Fiszdon JM, Johannesen JK, Lindenmayer J-P, McGurk SR, et al. Cognitive remediation for schizophrenia: An expert working group white paper on core techniques. Schizophr Res. janv 2020;215:49-53.

11. Wykes T, Huddy V, Cellard C, McGurk SR, Czobor P. A Meta-Analysis of Cognitive Remediation for Schizophrenia: Methodology and Effect Sizes. Am J Psychiatry. mai 2011;168(5):472-85.

12. Twamley EW, Burton CZ, Vella L. Compensatory Cognitive Training for Psychosis: Who Benefits? Who Stays in Treatment? Schizophr Bull. 1 sept 2011;37(suppl 2):S55-62.

13. Twamley EW, Vella L, Burton CZ, Heaton RK, Jeste DV. Compensatory Cognitive Training for Psychosis: Effects in a Randomized Controlled Trial. J Clin Psychiatry. 15 sept 2012;73(09):1212-9.

14. Allott K, van-der-EL K, Bryce S, Parrish EM, McGurk SR, Hetrick S, et al. Compensatory Interventions for Cognitive Impairments in Psychosis: A Systematic Review and Meta-Analysis. Schizophr Bull. 8 juill 2020;46(4):869-83.

15. Levaux M-N, Fonteneau B, Larøi F, Offerlin-Meyer I, Danion J-M, Van der Linden M. An Individualized and Everyday Life Approach to Cognitive Rehabilitation in Schizophrenia: A Case Illustration. Rehabil Res Pract. 2012;2012:1-9.

16. McGurk SR, Mueser KT, Covell NH, Cicerone KD, Drake RE, Silverstein SM, et al. Mental health system funding of cognitive enhancement interventions for schizophrenia: Summary and update of the New York Office of Mental Health expert panel and stakeholder meeting. Psychiatr Rehabil J. sept 2013;36(3):133-45.

17. Velligan; Mahurin RK; True JE; Lefton RS; Flores CV DI. Preliminary evaluation of cognitive adaptation training to compensate for cognitive deficits in schizophrenia. Psychiatr Serv. avr 1996;47(4):415-7.

18. Allott KA, Killackey E, Sun P, Brewer WJ, Velligan DI. Feasibility and acceptability of cognitive adaptation training for first-episode psychosis. Early Interv Psychiatry. déc 2016;10(6):476-84. 
19. Velligan DI, Prihoda TJ, Ritch JL, Maples N, Bow-Thomas CC, Dassori A. A Randomized Single-Blind Pilot Study of Compensatory Strategies in Schizophrenia Outpatients. Schizophr Bull. 1 janv 2002;28(2):283-92.

20. Velligan D, Diamond P, Maples N, Mintz J, Li X, Glahn D, et al. Comparing the efficacy of interventions that use environmental supports to improve outcomes in patients with schizophrenia. Schizophr Res. juill 2008;102(1-3):312-9.

21. Velligan DI, Weiden PJ, Sajatovic M, Scott J, Carpenter D, Ross R, et al. The expert consensus guideline series: adherence problems in patients with serious and persistent mental illness. J Clin Psychiatry. 2009;70 Suppl 4:1-46; quiz 47-8.

22. Kidd SA, Herman Y, Barbic S, Ganguli R, George TP, Hassan S, et al. Testing a modification of cognitive adaptation training: streamlining the model for broader implementation. Schizophr Res. juin 2014;156(1):46-50.

23. Kim EJ, Bahk Y-C, Oh H, Lee W-H, Lee J-S, Choi K-H. Current Status of Cognitive Remediation for Psychiatric Disorders: A Review. Front Psychiatry. 1 oct 2018;9:461.

24. Fisher C, Lindhorst H, Matthews T, Munroe DJ, Paulin D, Scott D. Nursing staff attitudes and behaviours regarding family presence in the hospital setting. J Adv Nurs. déc 2008;64(6):615-24.

25. Pilling S, Bebbington P, Kuipers E, Garety P, Geddes J, Orbach G, et al. Psychological treatments in schizophrenia: I. Meta-analysis of family intervention and cognitive behaviour therapy. Psychol Med [Internet]. juill 2002 [cité 8 avr 2021];32(05). Disponible sur: http://www.journals.cambridge.org/abstract_S0033291702005895

26. Allerby K, Sameby B, Brain C, Joas E, Quinlan P, Sjöström N, et al. Stigma and Burden Among Relatives of Persons With Schizophrenia: Results From the Swedish COAST Study. Psychiatr Serv. oct 2015;66(10):1020-6.

27. Friedman-Yakoobian MS, Mueser KT, Giuliano AJ, Goff DC, Seidman LJ. Family-directed cognitive adaptation pilot: Teaching cognitive adaptation to families of individuals with schizophrenia. Am $J$ Psychiatr Rehabil. 2 janv 2016;19(1):62-74.

28. Andersson G, Cuijpers P. Internet-Based and Other Computerized Psychological Treatments for Adult Depression: A Meta-Analysis. Cogn Behav Ther. déc 2009;38(4):196-205.

29. Cuijpers P, Cristea IA, Karyotaki E, Reijnders M, Huibers MJH. How effective are cognitive behavior therapies for major depression and anxiety disorders? A meta-analytic update of the evidence. World Psychiatry. oct 2016;15(3):245-58.

30. Olthuis JV, Watt MC, Bailey K, Hayden JA, Stewart SH. Therapist-supported Internet cognitive behavioural therapy for anxiety disorders in adults. Cochrane Common Mental Disorders Group, éditeur. Cochrane Database Syst Rev [Internet]. 12 mars 2016 [cité 8 avr 2021]; Disponible sur: http://doi.wiley.com/10.1002/14651858.CD011565.pub2

31. Ritterband LM, Thorndike FP, Ingersoll KS, Lord HR, Gonder-Frederick L, Frederick C, et al. Effect of a Web-Based Cognitive Behavior Therapy for Insomnia Intervention With 1-Year Follow-up: A Randomized Clinical Trial. JAMA Psychiatry. 1 janv 2017;74(1):68. 
32. Alvarez-Jimenez M, Alcazar-Corcoles MA, González-Blanch C, Bendall S, McGorry PD, Gleeson JF. Online, social media and mobile technologies for psychosis treatment: A systematic review on novel user-led interventions. Schizophr Res. juin 2014;156(1):96-106.

33. Westermann S, Rüegg N, Lüdtke T, Moritz S, Berger T. Internet-based self-help for psychosis: Findings from a randomized controlled trial. J Consult Clin Psychol. oct 2020;88(10):937-50.

34. Arnold C, Williams A, Thomas N. Engaging With a Web-Based Psychosocial Intervention for Psychosis: Qualitative Study of User Experiences. JMIR Ment Health. 19 juin 2020;7(6):e16730.

35. Gottlieb JD, Romeo KH, Penn DL, Mueser KT, Chiko BP. Web-based cognitive-behavioral therapy for auditory hallucinations in persons with psychosis: A pilot study. Schizophr Res. avr 2013;145(1-3):82-7.

36. Wasilewski MB, Stinson JN, Cameron JI. Web-based health interventions for family caregivers of elderly individuals: A Scoping Review. Int J Med Inf. juill 2017;103:109-38.

37. Harris AW, Kosic T, Xu J, Walker C, Gye W, Redoblado Hodge A. Web-Based Cognitive Remediation Improves Supported Employment Outcomes in Severe Mental Illness: Randomized Controlled Trial. JMIR Ment Health. 20 sept 2017;4(3):e30.

38. Newman MG, Szkodny LE, Llera SJ, Przeworski A. A review of technology-assisted self-help and minimal contact therapies for anxiety and depression: Is human contact necessary for therapeutic efficacy? Clin Psychol Rev. févr 2011;31(1):89-103.

39. de Bruin EJ, Meijer AM. The impact of online therapeutic feedback on outcome measures in InternetCBTI for adolescents with insomnia. Sleep Med. janv 2017;29:68-75.

40. Mol M, Dozeman E, Provoost S, van Schaik A, Riper H, Smit JH. Behind the Scenes of Online Therapeutic Feedback in Blended Therapy for Depression: Mixed-Methods Observational Study. J Med Internet Res. 3 mai 2018;20(5):e174.

41. Mohr S, Simon A, Favrod J, Fokianos C, Ferrero F. Validation de la version française du Profil des compétences de vie (Life Skills Profile) chez des personnes atteintes de schizophrénie. L'Encéphale. sept 2004;30(4):343-51.

42. Zarit SH, Reever KE, Bach-Peterson J. Relatives of the Impaired Elderly: Correlates of Feelings of Burden. The Gerontologist. 1 déc 1980;20(6):649-55.

43. Hébert R, Bravo G, Girouard D. Fidélité de la traduction française de trois instruments d'évaluation des aidants naturels de malades déments. Can J Aging Rev Can Vieil. 1993;12(3):324-37.

44. Auquier P, Simeoni MC, Sapin C, Reine G, Aghababian V, Cramer J, et al. Development and validation of a patient-based health-related quality of life questionnaire in schizophrenia: the S-QoL. Schizophr Res. sept 2003;63(1-2):137-49.

45. Lançon C, Aghababian V, Richieri R, Boyer L, Simenoni M-C, Auquier P. Insight et qualité de vie subjective chez les personnes souffrant de schizophrénie. Ann Méd-Psychol Rev Psychiatr. août 2011;169(7):429-31.

46. Kring AM, Gur RE, Blanchard JJ, Horan WP, Reise SP. The Clinical Assessment Interview for Negative Symptoms (CAINS): Final Development and Validation. Am J Psychiatry. févr 2013;170(2):165-72. 
47. Horan WP, Kring AM, Gur RE, Reise SP, Blanchard JJ. Development and psychometric validation of the Clinical Assessment Interview for Negative Symptoms (CAINS). Schizophr Res. nov 2011;132(2-3):140-5.

48. Misdrahi D, Verdoux H, Llorca P-M, Baylé F-J. [Therapeutic adherence and schizophrenia: the interest of the validation of the French translation of Medication Adherence Rating Scale (MARS)]. L'Encephale. août 2004;30(4):409-10.

49. Addington D, Addington J, Maticka-Tyndale E. Assessing depression in schizophrenia: the Calgary Depression Scale. Br J Psychiatry Suppl. déc 1993;(22):39-44.

50. Bernard D, Lançon C, Auquier P, Reine G, Addington D. Calgary Depression Scale for Schizophrenia: a study of the validity of a French-language version in a population of schizophrenic patients. Acta Psychiatr Scand. janv 1998;97(1):36-41.

51. Mackinnon A, Mulligan R. Estimation de l'intelligence prémorbide chez les francophones. L'Encéphale. févr 2005;31(1):31-43.

52. Delis DC, Freeland J, Kramer JH, Kaplan E. Integrating clinical assessment with cognitive neuroscience: Construct validation of the California Verbal Learning Test. J Consult Clin Psychol. févr 1988;56(1):123-30.

53. Wechsler, D. WAIS-IV Echelle d'intelligence de Wechsler pour adultes: Manuel d'administration et de cotation. ECPA. 2011.

54. Nelson H. A modified card sorting test sensitive to frontal-lobe defects. Cortex. 1976;(12):313-24.

55. Cardebat D, Doyon B, Puel M, Goulet P, Joanette Y. [Formal and semantic lexical evocation in normal subjects. Performance and dynamics of production as a function of sex, age and educational level]. Acta Neurol Belg. 1990;90(4):207-17.

56. Grace J, Stout JC, Malloy PF. Assessing Frontal Lobe Behavioral Syndromes with the Frontal Lobe Personality Scale. Assessment. sept 1999;6(3):269-84.

\section{Supplementary Files}

This is a list of supplementary files associated with this preprint. Click to download.

- Additionalfile1.doc 